\title{
The messy truth about drag
}

Richard Feynman once noted that scientific literature tends to present a distorted picture of research that is too linear and logical, as if researchers identify problems, devise theories and then test their ideas in experiment. In reality, of course, work often succeeds only by repeated failure and adjustment, and through lessons learned by accident. Scientists end up answering questions they had originally not dreamed of asking.

The same unnatural tidiness often afflicts the potted histories of textbooks, which offer students a version of the past cleansed of its natural chaos. Consider fluid dynamics, for example, and its 'simplest' problem - that of finding the drag on a sphere moving at fixed speed through a viscous fluid. Many texts give the impression that George Stokes worked out a good solution in 1851, at least for slow or 'creeping' flow (with Reynold's number, $R$, close to zero), finding that the drag coefficient goes as $C_{\mathrm{D}} \sim 1 / R$. Yet the truth about Stokes' solution turns out to be a little messier.

Physicists John Veysey II and Nigel Goldenfeld tell the surprising story (http://arXiv. org/abs/physics/0609138) of how it took more than 100 years before experiments could even measure the drag with appreciable accuracy, and 150 years of confusion before theorists learned how to calculate the drag, for a small Reynold's number, in a systematic and accurate way.

In many flows, a low value of the Reynold's number (defined in this case as $R=u_{\infty} a / v$, where $u_{\infty}$ is the sphere's velocity, $a$ its radius and $v$ the fluid viscosity) implies the dominance of viscous over inertial forces. But things aren't so simple in Stokes' problem, where the ratio of inertial to viscous forces is small near the sphere, but increases with distance $r$, scaling as Rr/a. For any positive value of $R$, no matter how small, viscous forces dominate near the sphere, but inertial forces do so in regions far away.

This makes it hard to proceed in the natural way, as Stokes attempted, by expanding around the $R=0$ solution, and it now seems that his famous result for the drag coefficient was roughly correct by fortuitous accident. When applied to a cylinder, rather than a sphere, his mathematical technique gives singularities. Later theorists - including Lord Rayleigh and Horace Lamb - pointed

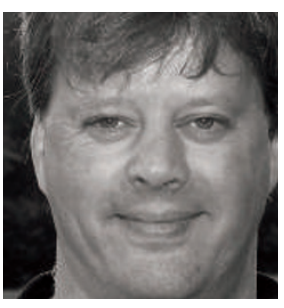

Stokes' famous

result was roughly correct by fortuitous accident.

\section{Expansion, and contraction}

By any quantitative measure you choose, the size of the scientific enterprise grew exponentially from its founding around 1700 until about 1970 . Thus, the cumulative number of scientific journals grew from a few around 1700 , to 10 around 1750,100 around 1800 and so on, reaching 100,000 around 1950. Similarly the number of physics PhDs in the United States grew from the first awarded in 1870 to about 10 per year in 1900,100 per year in 1930 and 1,000 per year around 1970; but then the growth stopped abruptly and the total has fluctuated around 1,000 per year ever since.

This sudden end of exponential expansion accounts for many of the difficulties we scientists have faced since then. The period 1950-1970 was a true golden age for American science. Young PhDs could choose among excellent jobs, and anyone with a decent scientific idea could be sure of getting funds to pursue it. The successes of science projects during the Second World War had paved the way for the federal government to assume responsibility for the support of basic research. Moreover, much of the rest of the world was still crippled by the after-effects of the war.

At the same time, the GI Bill of Rights sent a whole generation back to college, transforming the US from a nation of elite higher education to one of mass higher education. Before the war, $8 \%$ of Americans went to college, a figure comparable to that

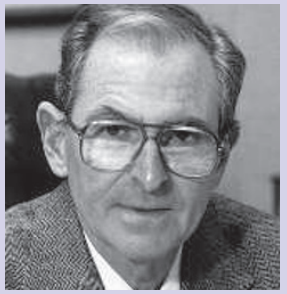

1950-1970

was a true golden age for American science. out why Stokes' approximations broke down and recognized the importance of the boundary layer. Yet incredibly, only in the 1950s did physicists finally produce the first legitimate approximate solution to lowest order in $R$.

Today, it may not be surprising to learn that Stokes' problem is yet another that turns out to be ripe for powerful renormalization group methods. Whereas the older results were only useful for infinitesimal values of $R$, modern work in this vein has produced natural results for the drag, accurate even for values of $R$ approaching one.

This would seem to bring to an end, temporarily at least, a long period of confusion. As Veysey and Goldenfeld note, their historical survey uncovered "over 150 years of diffuse, confusing, and sometimes contradictory experimental, numerical, and theoretical results". Hardly the logical scientific progress depicted in the textbooks.

On how science works, I think I prefer the analogy made by the American chemist Homer Adkins. "Basic research," as he put it, "is like shooting an arrow into the air, and, where it lands, painting a target”.

Mark Buchanan in France or the UK; now, more than half of all Americans receive some sort of post-secondary education. The expanding academic world of 1950-1970 created posts for the exploding number of new science PhDs, whose research led to the founding of journals, the acquisition of prizes and awards, and increases in every other measure of the size and quality of science.

But around 1970 there was a phase transition, all of those factors turned back on themselves. The golden era of exponential expansion had come to an end. We will all have to wrestle with the consequences of that transition for the foreseeable future. David Goodstein 